\title{
Focal Points and Bargaining ${ }^{1}$
}

\author{
Ken Binmore, Joe Swierzbinski, Steven Hsu and Chris Proulx \\ University of Michigan, Ann Arbor, MI 48109, USA
}

Abstract: This is a contribution to the growing experimental literature on how trial-and-error adjustment processes can establish a convention for coordination on an equilibrium in a game. A simple bargaining game introduced by Nash is used for this purpose. Subjects are conditioned in different treatments to use four different bargaining solutions. The stability of the conditioning is then studied as the bargaining game is played over an extended period. The data obtained is unusually sharp. In the long run, the median subject behaves as though optimizing often down to a fraction of a penny. The results are therefore not supportive of the view that strategic considerations in such situations can be neglected in favor of a study of fairness norms. Indeed, the equilibrium actually achieved in a session turns out to be a very good predictor of what the median subject says is "fair" in the game after play is over.

Early mankind soon reached the grand generalization that everything has its price, everything can be paid for. Here we have the oldest and most naive moral canon of justice, of all "fair play", "good will", and "objectivity". Justice at this level is good will operating among men of roughly equal power, their readiness to come to terms with one another, to strike a compromise $\cdots$

Friedrich Nietzsche, The Generalogy of Morals

\section{Introduction}

If I wait in the coffee shop for my wife while she searches for me in the car park, then we are experiencing a coordination failure. Schelling's [16] well known essay on coordination stresses the importance of focal points in such a context. The side of the road on which people drive is the standard example. Before any legislation appeared, it became focal to drive on the left in England, but to drive on the right in France.

Although the idea of a focal point is of great practical importance, the manner in which focal points become established and survive after their establishment re-

${ }^{1}$ We are grateful to the National Science Foundation for funding the experiments reported in this paper under Grant NSF-SES-8821521. We also gratefully acknowledge funding from the University of Michigan to set up the Michigan Economics Laboratory, where the experiments were conducted. We would also like to thank Richard Stallman and the Free Software Foundation for developing EMACS, Luke Tierney for developing LISPSTAT, and Hal Varian for showing us how to use both. 
mains a mystery. No consensus even exists about how this mystery should be investigated. Some authors emphasize rationality considerations to the exclusion of all else. However, it seems doubtful that the equilibrium selection problem of game theory is likely to be solvable by a technique that ignores what may be common knowledge among the players about the social norms of their culture. At the other extreme are authors who argue that social norms are so important that strategic issues can be neglected altogether. This view tends to be expressed most forcefully when the coordination problem is framed in a bargaining context. Fairness norms then enter the picture - and there is much evidence that such norms often do take precedence over strategic considerations in determining the behavior of subjects in certain types of laboratory experiments.

The story is further confused by the fact that those who emphasize rationality do not argue that real people are likely to find their way to what rationality supposedly recommends simply by thinking about the problem. Where pregame, cheaptalk sessions are not possible, the claim is that people will be able to find their way to the rational solution by trial-and-error if given long enough to gain experience of the game's strategic realities and the behavior of other members of the game-playing population. Nor are the views of those who emphasize social norms free from complication. They argue that several distinct social norms may compete for attention in certain contexts. So how do people decide which social norm should be honored?

This paper seeks to investigate such questions experimentally, using an archetypal example of a focal point problem. The example studied is the Nash [10] bargaining problem in which two players can achieve any point $x$ in a given feasible set $X$ provided that they can reach agreement. If they cannot agree, the result is a fixed disagreement point $\xi$ in the set $X$. Although the paper can be seen as a contribution to the expanding literature on experimental bargaining games, its potential applications to bargaining theory were a secondary consideration in our choosing the Nash bargaining problem for study. The primary reasons are twofold:

1. The literature contains numerous rival candidates as focal points in the Nash bargaining problem. We consider the Nash [10] Bargaining Solution, the Kalai-Smorodinsky [8] Bargaining Solution, the Utilitarian Solution associated with Harsanyi [5] and the Equal Increments Solution ${ }^{2}$ associated with Rawls [14].

2. Unlike the situations studied in the related work of Cooper et al. [3], and Van Huyck et al. $[17,18]$, the Nash bargaining problem has a continuous strategy space. In a discrete problem, it may be hard to destabilize an estab lished focal point. A population, for example, cannot gradually drift from driving on the left to driving on the right.

In order to study the Nash bargaining problem, it is necessary to say what the players need to do to reach an agreement. The most primitive mechanism is represented by the Nash [10] demand game and so we use this. In our experiment, a population of subjects play the same Nash demand game repeatedly, half the time as player I and half the time as player II, switching partners unpredictably after each

2 The Pareto efficient point $x \in X$ with $x_{1}-\xi_{1}=x_{2}-\xi_{2}$. 
play. At each play, a subject currently in the role of player I makes a demand $x_{1}$ and a subject in the role of player II makes a demand $x_{2}$. Each subject makes his demand in ignorance of the current demand of the other subject. (However, in our experiment, much information about past plays of the population as a whole was made available using a graphic display to be described later.) If the point $x=\left(x_{1}, x_{2}\right)$ of a partnered pair of subjects proves to be feasible, each receives his or her demand. Otherwise each receives the disagreement payoff. In our case, this was always zero (i.e. $\xi=0$ ).

Since conventional wisdom holds that the outcome in such a game depends on the players' attitudes toward risk, it is important to control for risk aversion. The standard technique is to pay subjects off in lottery tickets. In our case, the subjects had the opportunity to win $\$ 10$ with a probability equal to the number of lottery tickets they had accumulated divided by 100 . Rational agents would then be induced to behave as though they were risk neutral. To further impress the subjects with the importance of risk, lotteries were also introduced into each play of the game by fuzzing the boundary of the feasible set $X$. That is to say, the boundary of $X$ was expanded into a narrow strip. The feasibility of pairs of demands falling in this strip was uncertain. If, for example, the pair of demands fell on an $80 \%$ contour running through the strip, it would be found feasible by the computer with probability 0.8 .

We had a secondary motive in fuzzing the boundary which needs to be mentioned at an early stage because the fuzzing was instrumental in generating results that took us by surprise. This was a desire to be faithful to Nash's [10] original conception. He transferred his attention from the original Nash demand game to a version with a fuzzed or smoothed boundary because, without the smoothing, any individually rational, Pareto efficient $x$ in $X$ is a Nash equilibrium for the Nash demand game. With the smoothing, all Nash equilibria approximate the Nash Bargaining Solution. (See papers 4 and 8 of Binmore and Dasgupta [1].)

To see why such smoothing might help in responding to comment on the possible results of the experiment, consider what conclusions might be drawn if the subjects were to coordinate on the Utilitarian Solution. Harsanyi [5] might argue that such subjects were motivated by moral considerations. A welfare economist might argue that the explanation is that the Utilitarian Solution is Pareto efficient for the population as a whole over the course of the experiment. However, a game theorist of the variety that believes that thinking alone is adequate to get subjects immediately to the "right" Nash equilibrium would not be willing to admit that such normative considerations were necessarily relevant if the boundary of $X$ were not fuzzed. He could simply point to the fact that the Utilitarian Solution is a Nash equilibrium like every other individually rational, Pareto efficient outcome of $X$.

Although we prepared defenses in advance against certain potential criticisms by paying subjects off in lottery tickets (rather than directly in money) and by fuzzing the boundary of the feasible set, we did not seriously anticipate that these refinements would have any impact on our results. Our guess was that subjects are effectively risk neutral already with respect to the small sums of money we are able to pay. Moreover, after the fuzzing of the boundary of the feasible set, all the focal points we considered were within 0.1 of an $\varepsilon$-equilibrium with $\varepsilon=0.1$. It did not occur to us at the outset that subjects would be interested in discriminating at the 0.1 level, since 0.1 of a lottery ticket was worth only about a penny. However, our guess 
about the level at which subjects would choose to discriminate turned out to be badly wrong, and the results are perhaps more interesting than would have been the case if we had guessed right. To see why, it is necessary to continue outlining the design of the experiment.

After a hands-on interactive session at their computer to learn the mechanics of the program, the subjects first played ten "practice" games "against the computer". Both when playing the computer and when playing real opponents, subjects sometimes occupied the role of player I and sometimes the role of player II. Throughout the experiment, the subjects were shown the last demands made by all of their potential partners, both when the potential partner was player I and when he or she was player II. In the "practice games" this information display was used in a (successful) attempt to condition the subjects to begin the games against real opponents at one of the four "focal points" that we chose to study. For example, in the treatment designed to study the Equal Increments Solution as a possible focal point, the simulated potential partners that the subjects faced during the ten "practice games" were designed to converge slowly from a fixed initial configuration towards the Equal Incremenents Solution. After being conditioned to begin by making demands at or near the Equal Increments Solution, the question was then whether the subjects would continue to use this focal point once play against real opponents began.

We would not have been surprised to find that all the focal points we studied were stable. We thought the Utilitarian Solution might be particularly attractive. However, this proved to be the hardest to condition for in our main experiment. But the chief conclusion to which we were led by the data swept aside our initial expectations. We found a very strong tendency of the median subject to optimize relentlessly. Indeed, the extent of the optimization is almost absurd, since the subjects seem to have been sensitive to payoff differences right down to 0.01 of a lottery ticket (about one tenth of a penny). It is important to understand that the subjects were provided with graphic aids that made this possible for them to do reasonably efficiently if they so chose. In particular, they were provided with a display that allowed them to zero-in on the demand that would maximize their expected number of lottery tickets in the current game if their potential partners were to behave as they did the last time that they occupied their current role. Nevertheless, we were taken aback to find it necessary to compute the exact Nash equilibria of the discrete game that the digital character of computer technology made it necessary to present to the subjects in place of the continuous version that we had in mind as our basic model.

In our experiment, this required looking at a $100 \times 50$ payoff matrix in which each player's pure strategies consist of all possible locations for the cursor that he or she used in specifying what demand to make. This moved in steps of 0.1 of a lottery ticket. Each lottery ticket increases the probability of winning $\$ 10$ by 0.01 . Tus 0.1 of a lottery ticket corresponds to about one penny.

Figure 1 is intended to illustrate the main conclusions (Case 1) reported in this paper. The region shown represents the feasible set $X$ in each repetition of the Nash demand game. (The boundary shown is the $100 \%$ probability contour.) Coordinates are given as numbers of lottery tickets. The Equal Increments Solution, the KalaiSmorodinsky Solution, the Nash Bargaining Solution and the Utilitarian Solution are indicated with the letters $E, K, N$ and $U$ respectively. The box contains all Nash 

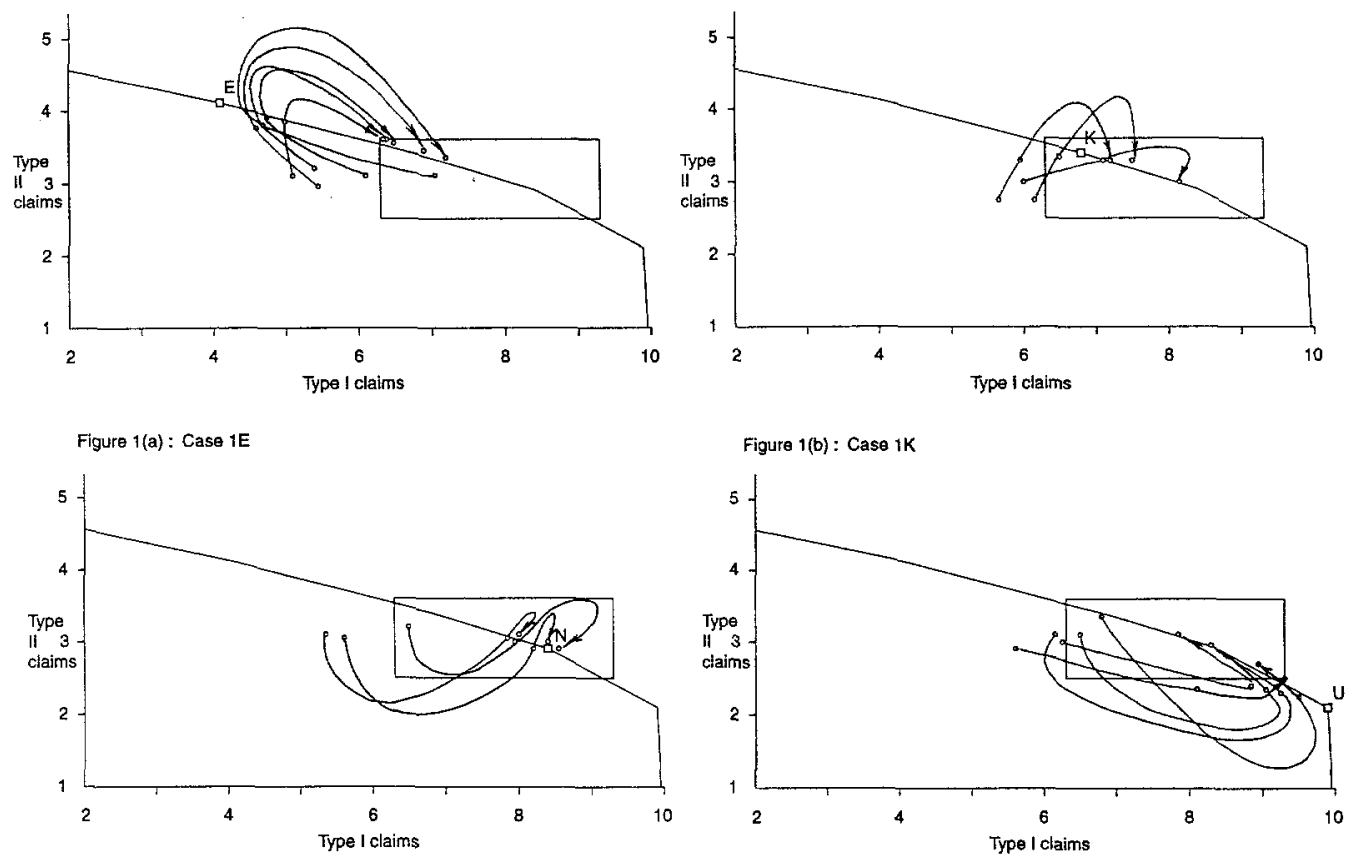

Figure $1(\mathrm{c})$ : Case iN

Figure 1(d) : Case 1U

Fig. 1. Summary of results in Case 1

equilibria of the discrete game. (Any Nash equilibrium of the continuous version approximates $N$.)

The arrows in Figure 1 do not indicate trajectories. Each of the 16 sets of arrows corresponds to a different group of 12 subjects and summarizes their experience by linking three points. Each point is a pair of median ${ }^{3}$ demands. The $x$-coordinate is the median demand of player Is. The $y$-coordinate is the median demand of player IIs. The three stages of the experiment at which these numbers are reported in the figure are:

1. At the very beginning - i.e. in the first and second practice games before any experience had been gained ${ }^{4}$.

2. Immediately after the practice games - i.e. in the 11 th and 12 th plays that followed the 10 conditioning practice games.

3. At the very end - i.e. in the 49 th and 50 th plays, after 10 practice games and 40 real games.

Three preliminary conclusions are listed below:

${ }^{3}$ The median of a set of numbers with an even number of elements is the mean of the two middle numbers.

${ }^{4}$ The computer updated its display every second play. 
- Whatever social norms the subjects may bring into the laboratory are easily erasable in the circumstances of the experiment. The subjects can be conditioned to begin playing for real close to any of the four focal points $E, K, N$, or $U$.

- The focal points $E$ and $U$ are not stable.

- The explanation that groups of subjects converge on an exact Nash equilibrium of the discrete game that they actually played fits the data very well.

It is natural to ask whether the Kalai-Smorodinsky solution $K$ would have been stable if it had not been included in the Nash equilibrium box. (One cannot, of course, exclude the Nash Bargaining Solution from the box.) One might also ask how the subjects would perform if not assisted with such a helpful graphic display. It is also interesting to know how the subjects responded to questions about "fairness" after playing the game.

The first question is not easily answered because it is difficult to adapt our experimental design to separate the Kalai-Smorodinsky Solution and the Nash Bargaining Solution adequately. Nevertheless, we hope to investigate this question with experiments that use a revised feasible set and to report any interesting results in a subsequent paper.

The extent to which our graphic display was essential to the results was explored in Case 2. Case $1 \mathrm{E}$ was modified so that the subjects were deprived of expected utility information in the graphic display. (Case 2 was otherwise identical to Case 1E.) Perhaps surprisingly, the subjects' behavior was not very different from that when the expected utility information was provided. Even the amount of variance in the data was only slightly higher. However, we only gathered data on this issue in the case when subjects were conditioned on the Equal Increments Solution.

Finally, the median of the claims reported as fair by each set of subjects after the experiment turned out to be closely correlated with the median of the claims actually made at the end of the experiment in which the subjects had participated. Very similar results were reported in Binmore et al. [2]. Perhaps we are therefore learning something about the origin of "fairness" norms.

\section{Theory}

This section begins by describing the ideas from cooperative bargaining theory used to locate the focal points in the experiment. Roth [15] discusses the properties and axiomatic characterization of the concepts. The remainder of the section briefly examines the problem of computing Nash equilibria in the smoothed Nash demand game. 


\subsection{Cooperative Solution Concepts}

The unsmoothed feasible set $X$ shown in Figure 2 is the convex hull of the points $(0,0),(10,0),(0,5),(4.1,4.1),(6.8,3.4),(8.4,2.9)$ and $(9.9,2.1)$. (In the smoothed case, a belt of fuzz surrounds $X$ as described in Section 2.2 and Section 3.) The shape of $X$ was determined by the need to separate the focal points $E, K, N$, and $U$ from each other. These four focal points are denoted by small boxes and labeled by letter in Figures 1, 2, and 4. The disagreement point $\xi$ is always $(0,0)$.

Equal Increments Solution $E$. This is a special case of a proportional bargaining solution as studied by Raiffa [12], Isbell [6], Kalai [7], Myerson [9], Roth [15], Peters [11] and others. Like the Utilitarian Solution to be considered shortly, the Equal Increments Solution requires that there be some basis for interpersonal comparison of utilities. This was provided in our experiments by the fact that the subjects alternated roles and were paid off in equally valuable lottery tickets both as player I and as player II. This same feature would also seem to justify restricting attention to cooperative bargaining solutions whose characterizations include a symmetry axiom. A proportional bargaining solution places the bargaining outcome at the Pareto efficient point of $X$ that lies on a line of fixed positive slope through the disagreement point $\xi$. The slope of this line equals the rate at which player I's utils are to be compared with player II's. With a symmetry axiom, this rate is 1 and so the relevant line through $\xi$ has slope 1 . The Equal Increments Solution therefore awards each player the same increment on his or her disagreement payoff. For the bargaining set described above, the Equal Increments Solution is $E=(4.1,4.1)$. It is worthwhile noting that $E$ is the point of $X$ that will be selected by Rawls' [14] maximin criterion.

Kalai-Smorodinsky Solution K. Kalai and Smorodinsky [8] offer this solution as an alternative to the Nash Bargaining Solution. Neither of these solutions depends on

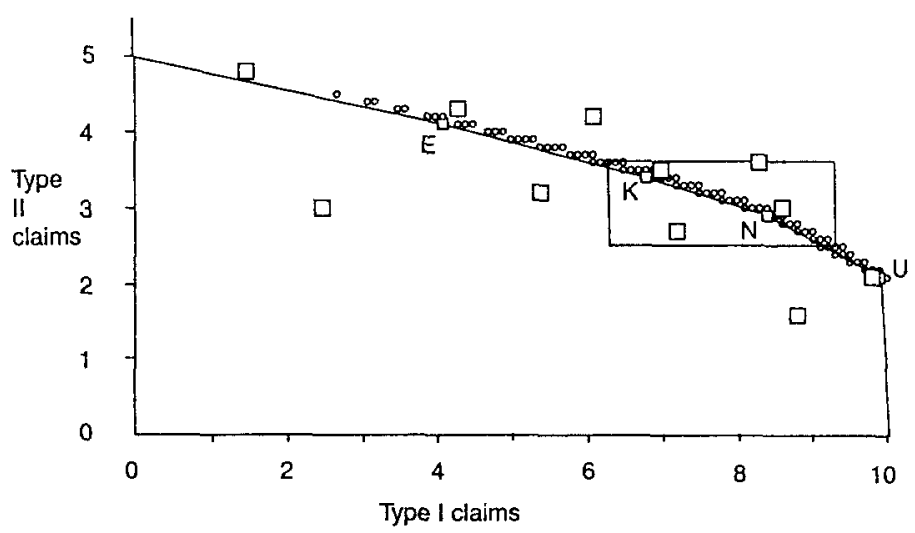

Fig. 2. The feasible set, focal points, the exact Nash eqilibrium box, $\varepsilon$-equilibria of the discrete game with $\varepsilon=0.1$, and initial robot claims 
interpersonal comparisons of utility. The Kalai-Smorodinsky Solution is found in our case by first locating the (infeasible) "utopian point" $(10,5)$ at which each player gets his or her maximum possible demand. A straight line is then drawn joining the disagreement point $\xi$ and the utopian point. The Kalai-Smorodinsky Solution $K=(6.8,3.4)$ is the Pareto efficient point of $X$ on this line.

Nash Bargaining Solution $N$. Nash [10] characterized $N=(8.4,2.9)$ as the value of $x$ in $X$ satisfying $x \geq \xi$ at which the Nash product $\left(x_{1}-\xi_{1}\right)\left(x_{2}-\xi_{2}\right)$ is maximized.

Utilitarian Solution $U$. The Utilitarian Solution $U=(9.9,2.1)$ is the value of $x$ in $X$ at which $x_{1}+x_{2}$ is maximized. Harsanyi [5] discusses its merits.

\subsection{Nash Equilibria in the Smoothed Game}

The details of how the Nash Demand Game was smoothed for the experiment are now described. The continuous case is described first and then the discrete approximation.

\section{The Continuous Version}

The smoothed version of the Nash Demand Game was obtained by making some of the demand pairs $x=\left(x_{1}, x_{2}\right)$ outside $X$ available with a specified probability $p(x)$. If the polar coordinates of $x$ are $(r, \theta)$, and $(R, \theta)$ is on the Pareto boundary of $X$, then

$p(x)=1-\left\{\left(1-\frac{r}{R}\right) / \gamma\right\}^{2}(R \leq r \leq(1+\gamma) R)$.

If $r<R$, then $p(x)=1$. If $r>(1+\gamma) R$, then $p(x)=0$. Notice that $\partial p / \partial r=0$ when $r=R$, so that probabilities change smoothly across the outermost $100 \%$ probability contour. Notice also that the region in which it is uncertain whether a particular demand pair $x$ is available shrinks to nothing when $\gamma \rightarrow 0$. In the experimental implementation, we took $\gamma=0.1$.

Binmore [1, p. 65] studies the reaction curves of the two players in smoothed demand games, and confirms Nash's claim that all nontrivial ${ }^{5}$ Nash equilibria converge on the Nash bargaining solution (under mild conditions) in the limiting case when $\gamma \rightarrow 0$. (The case of a piecewise linear boundary is not substantially different from the case when $X$ has a smooth boundary, which can be treated very easily as in Binmore [1, p. 159].)

With our choice of the function $p$, the reaction curves cross at just one point when $\gamma>0$. It follows that there is always a unique Nash equilibrium in the continuous case when $\gamma>0$. However, since the reaction curves are trapped in the region where $0<p<1$, they get very close together when $\gamma$ becomes small. In fact, given

${ }^{5}$ Excluding those equilibria in which both players make demands that are too large to be feasible whatever the other player may demand. 
any $\varepsilon>0$, we can make any individually rational, Pareto efficient $x$ in $X$ an $\varepsilon$-equilibrium by taking $\gamma$ sufficiently small.

The Discrete Version

We restricted players to making demands in multiples of 0.1 of a lottery ticket in the belief that we were thereby approximating the continuous version sufficiently closely for practical purposes. Figure 3 shows the reaction curves for the resulting discrete game.

Notice that the reaction curves in Figure 3 are very close together where things matter. Thus many points along the boundary are $\varepsilon$-equilibria for small values of $\varepsilon$. Note also that the reaction curves actually overlap over some of their range. Thus there are multiple exact Nash equilibria.

The small circles along the boundary in Figure 2 indicate all the nontrivial $\varepsilon$ equilibria with $\varepsilon=0.1$. There are 83 such equilibria some of which are also $\varepsilon$-equilibria for smaller values of $\varepsilon$. These $\varepsilon$-equilibria indicate a long and narrow region of relative stability where the gains from a unilateral deviation are small. There are 12 nontrivial exact Nash equilibria of the discrete game: $(6.3,3.6),(6.7,3.5),(7.0,3.4)$, $(7.3,3.3),(7.6,3.2),(8.0,3.1),(8.3,3.0),(8.5,2.9),(8.7,2.8),(8.9,2.7),(9.1,2.6)$, and $(9.3,2.5)$. These equilibria are contained in the large rectangular box shown in Figure 2 (and also in Figures 1, 4, and A1). This is the smallest box that contains all 12 equilibria.

Near the boundary of the feasible set, small changes in position can have large effects. For example, the point $(4.7,4.0)$ is an $\varepsilon$-equilibrium for $\varepsilon=0.05$. However, at the nearby point $(4.7,3.8)$, a type I player can increase his expected payoff by more than 0.7 lottery tickets by deviating from the choice 4.7 when his counterpart chooses 3.8.

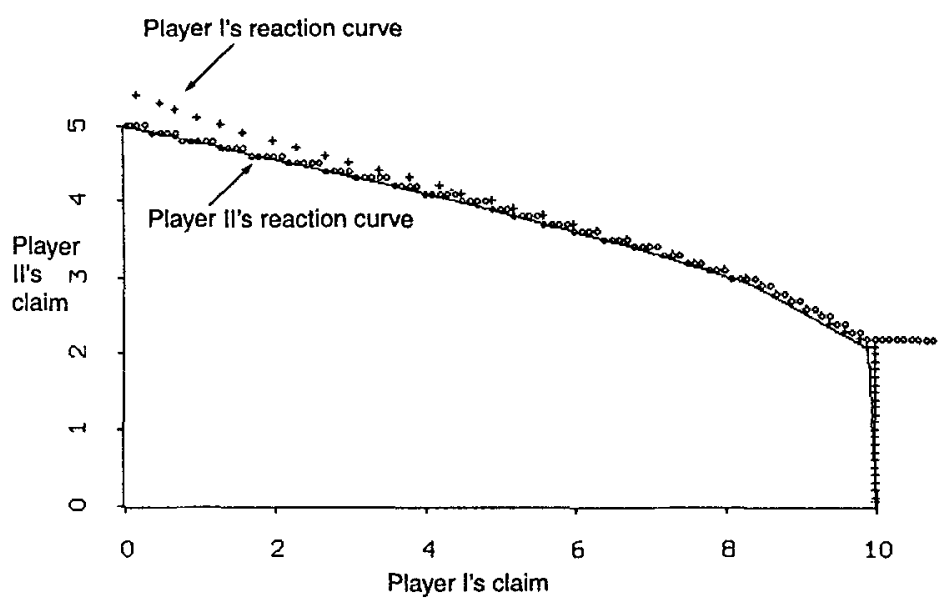

Fig. 3. The reaction curves for the discrete game 


\section{Practice}

The experiment was conducted at the Michigan Economics Laboratory using undergraduates of the University of Michigan. The subjects were recruited directly from classes rather than from a list of participants in previous experiments. Each subject was given a specific time to appear at the laboratory ${ }^{6}$ and promised $\$ 2$ for turning up on time, together with:

... the opportunity to win substantially more depending on how well you bargain and the circumstances in which you are placed. On average, subjects go away with between $\$ 10$ and $\$ 30$, but you might end up with more or less.

Each experimental session used 12 subjects who sat at networked microcomputers that were screened from each other. After reading the written instructions given as Appendix A1, the subjects participated in an interactive demonstration with the computer that was designed to familiarize them with how lottery tickets are converted into money and how demands are made and converted into lottery tickets.

Recall that, after each 10 games, a subject who has accumulated $N$ lottery tickets in these games, wins $\$ 10$ with probability $N \%$. This was operationalized by showing a "roulette wheel" split into a green winning region and a red losing region. A small yellow "ball" ran round the circumference of the wheel making appropriate noises, finally stopping in either the green or the red region. Where it stopped was fixed in advance only in the two demonstrations of its operation. Otherwise, everything advertized as random was indeed random.

The feasible set $X$ was shown as a blue region relative to white Cartesian coordinate axes against a black background. The fuzzy boundary was indicated as a halo shading gradually into black. An artist with a trained eye would perhaps have been able to deduce the probability that a demand pair in the halo would be accepted from the shade of blue at that point. Recall that the subjects occupied the role of player I about half the time ${ }^{7}$. They were therefore given experience of both roles.

Player I made demands by moving a cursor along the $x$-axis. The cursor was accompanied by a vertical line. After practising moving the cursor, a subject was shown the payoffs that he or she might receive for various demands that player II might make:

1. First a horizontal line appeared that intersected player I's vertical line well inside the blue feasible region. The point of intersection was then indicated with a flashing white circle. It was explained that each player gets his or her demand for certain.

2. Next a horizontal line appeared so that the point of intersection lay well inside the black region. The point of intersection was indicated with a flashing red circle. It was explained that each player gets nothing for certain.

\footnotetext{
${ }^{6}$ We did not want friends together in the same session.

${ }^{7}$ They did not strictly alternate roles since then they would not get the chance to play all other subjects. But they never occupied the same role three times in succession.
} 
3. Finally a horizontal line appeared so that the point of intersection lay in the fuzzy boundary region on the $80 \%$ probability contour. The point of intersection was indicated with a flashing white circle and the $80 \%$ probability contour was shown in white. It was explained that both players would get their demands $80 \%$ of the time and both players would get nothing $20 \%$ of the time.

4. Subjects received similar information after each practice and real game. After subjects registered their demands, a line was added to each subject's display indicating the demand of the opponent with whom he or she had been matched. If the intersection of the two demands was well inside the feasible set, the message "These claims are always acceptable. You both get your claims." was displayed. If the intersection was well outside the feasible set, the message "These claims are never acceptable. You both get nothing." was shown.

If the intersection fell near the boundary, for example on the $65 \%$ contour, then the computer displayed the message "These claims are acceptable $65 \%$ of the time. The computer accepts/rejects this pair of claims." The word "accepts" was shown in white alternating with the word "rejects" in red, the former remaining on the screen $65 \%$ of the time. Clicking sounds accompanied the changes in the words displayed. Eventually the alternation stopped and the subjects were informed whether or not they had received their demands on that particular occasion.

After practising in player I's role, each subject went through a similar experience in the role of player II. Note that we were anxious not to suggest any focal points at this stage, and so it was always left to the subject to choose where to place his or her demand cursor during the demonstration. Moreover, when the computer simulated an opponent, its placing of the opponent's cursor was made a function of the placing of the subject's cursor.

The next step in the demonstration was to teach the subjects to understand the information about their potential opponents that would be supplied. They were shown a screen with small yellow squares superimposed on the blue feasible region. It was explained that each yellow square represents one of the other subjects, each of whom is equally likely to be your next opponent. The $x$-coordinate of the center of a square represents the demand that the subject represented by that square last made when occupying the role of player I. The $y$-coordinate represents the demand that he or she last made when occupying the role of player II. The computer moved player I's cursor back and forward to show how a yellow square becomes red as the demand represented by the current placing of player I's cursor becomes incompatible with the demand last made by the subject represented by that square when occupying the role of player II. When there is only a probability $p$ of the demand pair being incompatible, only a fraction $p$ of a yellow square becomes red. As player I's cursor is moved, the impression is therefore of a collection of small square vessels being slowly filled with blood.

Subjects in Case 2 were offered only this information about the other subjects. Subjects in the main experiment (Case 1) were offered more information. After the screens that explained the yellow squares, they were shown the same screens again 
with the addition of an "expected utility indicator" on the $x$-axis. This took the form of a second cursor that showed the expected number of lottery tickets that player I would receive if he or she made the demand indicated by the current placing of his or her demand cursor, and the other subjects made the demands indicated by the current placing of the yellow squares. The part of the $x$-axis between the origin and the second cursor was highlighted in yellow and the part of the $x$-axis between the second cursor and the demand cursor was highlighted in red. Trials with the equipment indicate that differences in expected utility as small as 0.025 lottery tickets could be detected with this indicator.

As the demand cursor moved, the second cursor acting as an "expected utility indicator" moved also. When it reached its maximum point, it left behind a third stationary cursor (like a max-min thermometer). It was therefore relatively easy for a subject so minded to locate the demand that maximized his or her expected utility on the myopic assumption that other subjects would play as they did last time.

After seeing the screens that described how information is presented to player I, the subjects were invited to move player I's demand cursor back and forward to see how the display changes as the cursor moves. They were then shown the whole thing over again from player II's viewpoint.

Those who have no experience of presenting information to subjects through interactive computer programs may feel that the subjects must have been overwhelmed by such a complicated demonstration. However, subjects seemed to have very little difficulty in absorbing the information offered. They almost never used the facility for calling the assistant to answer questions, and seldom reported any confusion about what was expected of them in the questionnaire that they completed after the experiment. Perhaps this is not so surprising, since everybody has experience of video games requiring the need to absorb far more information much more quickly.

After the demonstration, each subject played 10 "practice games" against "robot opponents". These practice games were not simply to familiarize the subjects with the way the games were played. The practice games were a deliberate attempt to condition the subjects to use one of the focal points on which the experiment concentrated. In each case, the subjects were told that, in the practice games, the yellow squares each represented a robot opponent that they might be playing. The initial distribution of robot squares is shown by the larger, unlabeled squares in Figure 2.

Case 1 was separated into four treatments. In Case $1 \mathrm{E}$, the robots were programmed to converge slowly on the Equal Increments Solution, $E$. In Case $1 \mathrm{~K}$, the robots converged on $K$, in Case $1 \mathrm{~N}$ on $N$, and in Case $1 \mathrm{U}$ on $U$. Case 2 was the same as Case 1E, but without the "expected utility indicator". Throughout the experiment, subjects' screens were updated with information about their potential opponents every second game. Note also that convergence was deliberately not total. The robots converged only to the extent that they arrived in the neighborhood of the selected focal point at the time of the last update during the practice games.

At the end of the ten practice games, subjects were shown the "roulette wheel" and discovered whether they would win or lose a prize for the practice session. They were forewarned that the prize for the practice games would be only $\$ 1$ instead of the $\$ 10$ prize that was at stake in each of the four sets of ten games that were subsequently to be played for real. 
In each game, subjects were matched at random with the constraints that no subject ever played the same opponent twice in succession or occupied the same player role three times in succession. The interaction between paired subjects was anonymous. Except for their opponent's current demand, subjects were not given any information about the identify of their partner.

After each game, subjects were shown a roulette wheel that exhibited how many lottery tickets they had accumulated since it was last "spun". At the end of each set of 10 games the roulette wheel was spun for a prize of $\$ 10$. Our strong impression is that such "intermittent reinforcement" does indeed quicken the interest of subjects (as psychologists report). However, perhaps more importantly, it also provides some mildly entertaining interludes in an experimental session, which although only half an hour or so long, could easily become very dull if not broken up into bite-size pieces.

After playing 50 games (10 for practice and 40 for real), the subjects were told how much money they had won, and asked to complete a brief computerized questionnaire before leaving. Finally, they were called to be paid off one by one with the aim of minimizing interaction between subjects as they left the vicinity of the laboratory.

\section{Results}

Recall that Case 1 was split into four treatments in which an attempt was made to condition subjects to use one of the four focal points $E, K, N$, or $U$ (by programming their robot opponents to converge on one of these focal points during the practice games). Only treatment $E$ was used in Case 2, which differed from Case $1 E$ only in that no "expected utility indicator" was provided.

In each case, 10 practice games were followed by 40 real games with pauses to spin a "roulette wheel" every tenth game. The experiment concluded with a brief computerized questionnaire.

\subsection{Case 1}

Figure 1 summarizes the overall picture. This shows the median demand pairs before the subjects had any experience, immediately after they had been conditioned by the practice games, and at the end of the experiment. All that need be added to the discussion of Figure 1 given in the Introduction is that the data better fits the hypothesis that the subjects were conditioned to play a best response to the robot opponents they faced rather than to play the focal point itself. This point is discussed further in Section 5 and is, of course, consistent with our overall explanation of the data: namely, that the story is one of relentless optimization by the median player. 
Table A1 of Appendix A2 gives the data from which Figure 1 was constructed together with the corresponding data for Case 2.

Figures A1(a) through A1(e) of Appendix A2 show some typical trajectories for median demand pairs over the entire 50 games. For the practice games, the trajectories show the median claims of both the subjects and their robot opponents. In practice games $1,4,6,7$, and 9 , the subjects made claims as player I while the computer made claims as player II. The roles were reversed in the other practice games. When interpreting the trajectories, it is helpful to keep in mind that the $x$-coordinate of the point labeled " 10 " represents robot rather than human claims. Table A1, on the other hand, reports the median human claims in games 9 and 10 . That is, Table A1 reports the median human claims as player I from game 9 and the median human claims as player II from game 10 .

Unlike Figures 1 and 4, each point in Figure A1 represents the median demands by type I and type II players in a single game rather than a pair of games. The numbers in Figure A1 indicate the games that correspond to various points. It was not possible to label the same set of games on each trajectory and keep the figures legible.

From the positions of points 9 and 10 on the trajectories for Case $1 \mathrm{U}$, one can see that the human claims in the last practice games are close to the utilitarian focal point but the trajectories quickly move away from the focal point in the first few real games. This pattern, which indicates the difficulty we encountered in conditioning subjects to use the utilitarian focal point $U$, was also observed in the other three trajectories for Case $1 \mathrm{U}$. (One could also turn to Table Al and, for each treatment, compare the median claims made by player I in games 9 and 10 with the median type I claims made in games 11 and 12.)

The extent of the variation in individual claims can also be assessed using Tables A1 and A3. The variation indicates that subjects did not always use the "expected utility indicator" with any precision, if at all. Such variation in behavior should not be dismissed as mere "noise". Computer simulations of myopic adjustment that we conducted before experimenting with real subjects exhibited much slower movement along the boundary away from a "focal point" than was observed in the experiment. We believe that the slow convergence of the simulated claims was due to the absence of variation in the simulation.

Other points that can be checked by examining Table A1 are the extent to which the subjects within each population group finally converged on the same claim and the number of games required for the median claims in each experiment to converge. In each experiment, there is little or no difference between the median of the claims in the last two games and the median of the claims in the last ten games. That is to say, the median claims had already converged by the 41 st game.

\subsection{Case 2}

This was an attempt to see how important the "expected utility indicator" is for the conclusions of Case 1. It was replication of Case $1 \mathrm{E}$ without the expected utility 
indicator. Figure 4 is the equivalent of Figure 1 for Case 2. The relevant data appears in Table A1 of Appendix A2. Figure A1(f) shows a typical trajectory.

The results are broadly similar to those reported in Case 1E. This came as yet another surprise. How did the subjects manage to behave as though they were optimizing when deprived of the means to do so with the accuracy that their behavior seems to indicate?

\subsection{Questionnaire}

In a previous paper (Binmore et al. [2]), a strong tendency was noted for people asked after the experiment for their views on what is "fair" to give answers that correlate with their experience of what actually happened in the bargaining game they had just played. We now briefly explore this issue. The relevant data is contained in Table $\mathrm{A} 1$ of Appendix $\mathrm{A} 2^{8}$.

For each experiment, Figure 5 plots against the median of the last claims made as player I the median claim said to be "fair" for player I in reply to the question:

What do you feel would be a fair amount for each player to get?

Move each player's cursor to the fair amount.

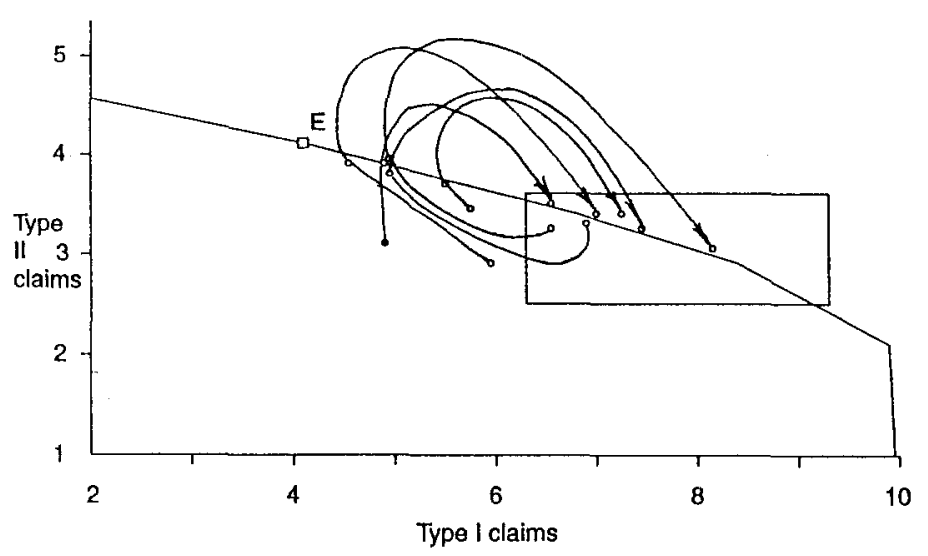

Fig. 4. Summary of results in case 2

${ }^{8}$ Because a subject could be player I or player II for two games in a row, the median claims in games 49 and 50 are almost but not quite identical to the median last claims. In experiment 17, the median of the last claims made as player I was 7.60 and in experiment 21 was 6.70 . In all other cases, the difference between the median claims in games 49 and 50 and the median last claims did not exceed 0.1 lottery ticket. Figure 5 was constructed using the median last claims rather than the median claims in games 49 and 50 . 


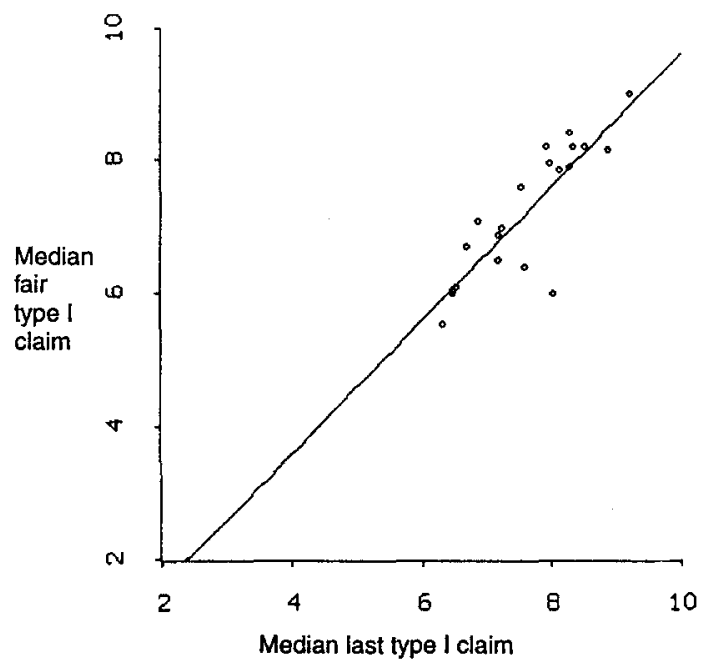

Fig. 5. Comparison of the medians of the last actual claims made by player I in each experiment with the medians of the claims said to be "fair" for player I

Notice the marked tendecy to report as fair what actually happened.

The line shown in Figure 5 is the least squares regression line obtained by regressing the median fair claims on the median last claims. It satisfies the equation: $y=-0.3970+0.9999 x$. The standard errors of the intercept and slope are 1.0748 and 0.1401 respectively and $R^{2}=0.7282$.

It is perhaps interesting that the points in Figure 5 whose residuals have the greatest absolute value are from experiments 17 and 18 both from Case 2 . If only data from Case 1 is used, the results are essentially the same except that the $R^{2}$ increases to 0.8977 . The results are also unaffected if the median last and median fair claims for player II are used instead of the claims for player I. In all cases, we obtain a highly significant relationship between the median fair claims and the median last claims and a regression line with an intercept close to 0 and a slope close to 1 .

If, for all 252 subjects, we regress the claim which each individual designated as fair for player I $(y)$ against the last claim made by that individual as player I $(x)$, then we obtain the equation: $y=2.2261+0.6382 x$ with standard errors 0.7699 and 0.1007 and $R^{2}=0.1385$. There is still a significant relationship, but the unexplained variation in the individual claims is much higher than for the median claims.

A later question asked:

Is this the sort of situation in which people ought to "play fair", or is it socially acceptable to use whatever bargaining power one has?

Of the 252 subjects who participated in the entire experiment, 89 subjects, approximately $35 \%$, said that one ought to "play fair". The rest said that it was acceptable to use one's bargaining power. 


\section{Statistics}

This section reports numerical summaries of the data that complement the graphical summaries in Figures 1 to 5 and Figure A1.

Recall that the subjects' information about the play of their potential opponents was updated every second game. In order to compare the effects of the different treatments in Case 1 and to study the stability of the focal points, we consider the subjects' claims in the last practice games, 9 and 10, the first real games, 11 and 12, and the last real games, 49 and 50. For each treatment, Table 1 reports information about the median claims in these pairs of games.

Table 1. For selected pairs of games, this table reports Euclidean and standardized measures of distance from the populations of median claims in each treatment to the appropriate focal points and to the median myopic best replies to the robot claims in games 9 and 10 . The table also reports the means of the maximum gains obtained by unilateral deviations from each pair in the designated sets of median claims. Table 1 uses the median claims reported in Table A1 and the maximum gains from a unilateral deviation reported in Table A3 as input

\begin{tabular}{|c|c|c|c|c|c|}
\hline Games & $\begin{array}{l}\text { Euclidean } \\
\text { distance }\end{array}$ & $\begin{array}{l}p \text {-value for } \\
\text { Hotelling's } \\
T^{2}\end{array}$ & $\begin{array}{l}\text { Euclidean } \\
\text { distance }\end{array}$ & $\begin{array}{l}p \text {-value for } \\
\text { Hotelling's } \\
T^{2}\end{array}$ & $\begin{array}{l}\text { Mean } \\
\text { maximum } \\
\text { gain from } \\
\text { a deviation }\end{array}$ \\
\hline \multicolumn{6}{|l|}{ Case 1, E } \\
\hline & \multicolumn{2}{|l|}{ Focal point } & \multicolumn{3}{|c|}{ Myopic best reply } \\
\hline $9 \& 10$ & 0.690 & 0.0057 & 0.210 & 0.2412 & 0.514 \\
\hline $11 \& 12$ & 0.708 & 0.0007 & 0.234 & 0.0065 & 0.609 \\
\hline $49 \& 50$ & 2.637 & lt. .0001 & 2.127 & 0.0002 & 0.011 \\
\hline \multicolumn{6}{|l|}{ Case $1, \mathrm{~K}$} \\
\hline & \multicolumn{2}{|l|}{ Focal point } & \multicolumn{3}{|c|}{ Myopic best reply } \\
\hline $9 \& 10$ & 0.261 & 0.1157 & 0.134 & 0.1316 & 0.584 \\
\hline $11 \& 12$ & 0.295 & 0.2675 & 0.589 & 0.2559 & 0.655 \\
\hline $49 \& 50$ & 0.841 & 0.3460 & 0.554 & 0.5756 & 0.048 \\
\hline \multicolumn{6}{|l|}{ Case $1, \mathrm{~N}$} \\
\hline & \multicolumn{2}{|l|}{ Focal point } & \multicolumn{3}{|c|}{ Myopic best reply } \\
\hline $9 \& 10$ & 0.237 & 0.1267 & 0.097 & 0.2712 & 0.254 \\
\hline $11 \& 12$ & 0.409 & 0.0378 & 0.201 & 0.0322 & 0.214 \\
\hline $49 \& 50$ & 0.130 & 0.2765 & 0.117 & 0.4508 & 0.018 \\
\hline \multicolumn{6}{|l|}{ Case $1, \mathrm{U}$} \\
\hline & \multicolumn{2}{|l|}{ Focal point } & \multicolumn{3}{|c|}{ Myopic best reply } \\
\hline $9 \& 10$ & 0.160 & 0.0576 & 0.117 & 0.1786 & 0.160 \\
\hline $11 \& 12$ & 0.977 & 0.0095 & 0.860 & 0.0480 & 0.564 \\
\hline $49 \& 50$ & 1.548 & 0.0015 & 1.413 & 0.0041 & 0.025 \\
\hline \multicolumn{6}{|l|}{ Case 2, E } \\
\hline & \multicolumn{2}{|l|}{ Focal point } & \multicolumn{3}{|c|}{ Myopic best reply } \\
\hline $9 \& 10$ & 0.620 & 0.0544 & 0.156 & 0.1451 & 0.379 \\
\hline $11 \& 12$ & 0.905 & 0.0315 & 0.399 & 0.1335 & 0.295 \\
\hline $49 \& 50$ & 3.274 & 0.0028 & 2.765 & 0.0053 & 0.036 \\
\hline
\end{tabular}


The data used to construct Table 1, as well as Figures 1, 4, and 5, is contained in Tables A1 and A3 of Appendix A2 and summarized in Table A2. For each experiment, Table A1 reports the median and the standard deviation of the populations of type I and type II claims in selected pairs of games. Table A1 also reports the median and standard deviation for the populations of claims in the last 10 games, 41 through 50, and for the populations of claims that were designated as "fair" for each type of player in the questionnaire at the end of the experiment.

We can regard each of the pairs of type I and type II median claims reported in Table A1 as a single data point. The replications of each treatment then provide a population of data points for each pair of games. Table A2 reports summary statistics for each of these populations. The statistics in Table 1 were also constructed by treating the pairs of median claims in Table A1 as two-dimensional data points.

The second column of Table 1 reports the Euclidean distance between the center of gravity of each population of median claims and the relevant focal point. (The coordinates of the centers of gravity are reported in columns 2 and 4 of Table A2 ${ }^{9}$.) These distances confirm the impression left by Figures 1 and 4 that, for each case and treatment, the distance between the population of medians in the last practice or first real games and the treatment's focal point is always small compared, for example, to the distance between adjacent focal points.

For Case 1U, the jump in the distance to the utilitarian focal point from the last practice to the first real games is an indication of the difficulty we had in conditioning subjects to begin play at the utilitarian point. Note also that the distance from the populations to their respective focal points increases substantially from the first to the last real games for Cases $1 \mathrm{E}, 1 \mathrm{U}$, and Case 2. The distance increases somewhat for Case $1 \mathrm{~K}$ and actually decreases for Case $1 \mathrm{~N}$. The change in the distance from the populations of medians to their focal points over the course of the real games is one index of the relative stability of the four focal points.

Is the distance between the center of gravity of each population and the relevant focal point small or large relative to the variation within the population? If the data were one-dimensional, the $t$-statistic could be used to measure the distance from the sample mean to the focal point in units of the estimated standard deviation of the sample mean. Column 3 of Table 1 measures distances using Hotelling's $T^{2}$ statistic which is a multidimensional generalization of the $t$-statistic ${ }^{10}$.

Just as the $t$-statistic can be used to construct confidence intervals around the sample mean, the two-dimensional $T^{2}$ statistic can be used to construct confidence ellipses around a sample's center of gravity. Rather than directly reporting the value of $T^{2}$, column 3 of Table 1 reports the $p$-value of the confidence ellipse that surrounds the center of gravity of each population of median claims and passes through the appropriate focal point.

Smaller $p$-values correspond to ellipses that are further from the center of gravity. Under the assumption that the population of median claims represents a sample drawn from a bivariate normal distribution, a $p$-value less than 0.05 implies that one

9 The $x$ and $y$ coordinates of the center of gravity of a cloud of two-dimensional points are the means of the $x$ and $y$ coordinates of the points in the cloud.

${ }^{10}$ See Rao [13] (or any good text on multivariate statistics) for a definition and discussion of the properties of Hotelling's $T^{2}$ statistic. 
can reject the hypothesis that the center of gravity of the true distribution is equal to the focal point with a level of confidence greater than $95 \%$. However, caution should be used in interpreting the standardized distances in this way, especially in light of the small numner of observations ( 3 or 5 ) in each sample.

If the median subject in the practice games of each experiment optimized myopically against his or her robot opponents, then the median claim in the last practice games would not necessarily be close to the focal point. Instead, this claim would be close to the myopic best reply to the population of robot opponents in games 9 and 10.

The population of robot opponents varied slightly from subject to subject within the same treatment. However, by games 9 and 10, the myopic best replies to the possible populations of robots within each treatment were virtually identical ${ }^{11}$. Let $E_{\text {br }}$ denote the ordered pair of medians of the myopic best replies by type I and type II players respectively to the robot populations in games 9 and 10 of Case 1E (and Case 2). $E_{\mathrm{br}}=(4.6,4.0)$. For Case $1 \mathrm{~K}, K_{\mathrm{br}}=(7.1,3.4)$. For Case $1 \mathrm{~N}, N_{\mathrm{br}}=(8.2,3.0)$. For Case $1 \mathrm{U}, U_{\mathrm{br}}=(9.8,2.2)$.

Columns 4 and 5 of Table 1 report the same statistics as columns 2 and 3 except that the distances measured are those between the centers of gravity of each population and the relevant median myopic best replies rather than the relevant focal points.

For Case 2 and each treatment of Case 1, the Euclidean distance from the center of gravity of the median claims in games 9 and 10 to the median myopic best reply is less than the distance to the corresponding focal point. In each case, the $p$-value measuring the standardized distance to the focal point is smaller (so the standardized distance is greater) than the $p$-value measuring the distance to the myopic best reply. If Hotelling's $T^{2}$ statistic were used as the basis of a hypothesis test, we would be unable to reject the hypothesis that the center of gravity of the true distribution of each sample of median claims in games 9 and 10 is the relevant myopic best reply. Statistical tests on the populations of individual subjects' claims in games 9 and 10 support a similar conclusion ${ }^{12}$.

"In other respects, the alternate distance measures in columns 3, 4, and 5 of Table 1 reinforce the information conveyed by the distances reported in column 2 .

${ }^{11}$ In the real games, subjects were shown the previous claims of each of their 11 possible opponents. However, in the practice games only 10 (out of a possible 11) robot squares were displayed at a time, and which squares were displayed varied randomly from subject to subject. By games 9 and 10, the 11 possible populations of robot opponents in each treatment were all very similar, so that this source of variation had almost no effect on the myopic best replies. For each treatment, the difference between the largest and the smallest myopic best reply for player I in games 9 and 10 was less than or equal to 0.2 lottery tickets. For best replies by player II, the difference was less than or equal to 0.1 lottery ticket.

12 A sign test was used to test the hypothesis that the median of the population of type I claims made by each subject in game 9 pooled across all replications of the same treatment was the same as the median of the myopic best replies to the populations of robot claims for that treatment. (See, for example, Gibbons and Chakraborti [4] for a description and analysis of the sign test.) For each treatment, we were unable to reject this hypothesis at the usual $5 \%$ level of significance. A similar test for the populations of type II claims in game 10 was unable to reject the hypothesis for each of the four populations of type II claims in Case 1. For the type II claims in Case 2, the sign test would reject the hypothesis at the $5 \%$ but not the $1 \%$ level of significance. 
For Case 1E, Case 1U, and Case 2, the Euclidean distance from the myopic best reply increases considerably from the first to the last pair of real games. The corresponding $p$-values for both the focal point and myopic best reply all decrease. The Euclidean distance to the myopic best reply in Case $1 \mathrm{~K}$ is relatively small and approximately the same in games 11 and 12 and games 49 and 50. The Euclidean distances to the best reply for Case $1 \mathrm{~N}$ are even smaller than for Case $1 \mathrm{~K}$ and the distance from the center of gravity in games 49 and 50 is less than the distance in games 11 and 12. All four $p$-values in games 11 and 12 and games 49 and 50 of Case $1 \mathrm{~K}$ are relatively large. The $p$-values for Case $1 \mathrm{~N}$ increase from the first to the last pair of real games ${ }^{13}$.

By comparing the median claims reported in Table $1 \mathrm{~A}$ for games 49 and 50 with the coordinates of the exact Nash equilibria reported in Section 2.2, one can confirm that the median claims in each experiment typically end up very close to one of the exact Nash equilibria of the discrete demand game. Table A3 of Appendix A2 further investigates the convergence of the subjects' claims to $\varepsilon$-equilibria of the discrete game.

For the designated games of each experiment, Table A3 reports the maximum expected gain that a player can achieve by deviating from the median claim for his type that was reported in Table A1 when the player expects his opponent to make the corresponding median claim that was reported for her type in Table A1. The reported gain is the maximum of that achievable by either type I or type II players. The last column in Table 1 reports the mean of the maximum expected gains in Table A3 for each series of experiments.

In every experiment, including those where there was no expected utility indicator, Table A3 shows that by the last real games the median subjects had found their way to $\varepsilon$-equilibria for remarkably small values of $\varepsilon$.

As indicated in Tables 1 and A3, at least one type of player could typically receive an expected gain of about 0.5 lottery tickets (or 1 nickel) by unilaterally deviating from the median claim in the first real games of an experiment. An expected gain of that size would have been easily observable with the expected utility indicator provided to subjects in Case 1. Moreover, an increase of 0.5 lottery tickets in each of 40 real games corresponds to a 20 percent greater chance of winning 10 dollars or an expected gain of 2 dollars. Such a gain, while not large, might not have been negligible in the eyes of the subjects. By the last real games of the experiment, the typical gain obtained by a unilateral deviation from the median claims had shrunk to about 0.03 lottery tickets (about $1 / 3$ of a penny or 12 cents over the course of 40 games).

The standard deviations reported in Table A1 show that not all subjects made claims close to the median especially in the early games of an experiment. The numbers reported in parentheses in Table A3 help assess the implications of this variability.

13 The correlation coefficients reported in Table A2 indicate that the populations of medians in games 49 and 50 are almost "one-dimensional". One might therefore wonder if, after all, a one-dimensional standardized distance might be more appropriate than one based on Hotelling's $T^{2}$ statistic. It turns out that $p$-values based on the $t$-statistic for the populations of median type I claims exhibit qualitative behavior which is similar to that of the $p$-values reported in Table 1. 
For each pair of games and each experiment, one can calculate the maximum expected gain that a player can achieve by deviating from a particular claim when the player's opponent chooses randomly from the 12 opposing claims actually made in these games. By calculating a maximum expected gain in this way for each of the the 24 type I and type II claims actually made in a particular pair of games, one obtains a population of 24 maximum expected gains from a deviation. The first number of each pair in parentheses in Table A3 is the median of such a population of maximum expected gains. The second number is the 90 th percentile of these gains.

The median expected gain defined in this way differs from the expected gain discussed earlier partly because in one case each claim is matched against a population of opposing claims while in the other case a claim is matched only against the single opposing median claim. Nevertheless, the two statistics behave similarly. In almost all experiments, the median expected gain decreases from the first to the last pair of real games. In addition, the median expected gain in the last real games is often less than 0.1 lottery ticket (that is less than 1 penny) and always less than 0.2 lottery tickets.

The 90th-percentile expected gains also typically decrease from the first to the last pair of real games in each experiment. Part of this decrease is probably due to the tightening of the distributions of claims around their medians which is also shown by the changes in the standard deviations reported in Table A1. However, the magnitudes of the 90th-percentile expected gains are also noteworthy. In a number of experiments, this percentile is greater than 0.5 lottery tickets even in the last pair of real games. This supports the conclusion stated earlier that, although the median subject may optimize relentlessly, this is not necessarily true of every subject in every game.

\section{Conclusions}

The results of this series of experiments provide no comfort for those who argue that strategic considerations have little relevance to how people resolve coordination problems. If people are equipped with social norms that are relevant to the problem faced by our subjects, then it seems that a small amount of conditioning is sufficient to displace them in favor of a focal point of the experimenter's choice. Thereafter the median subject seems to optimize insofar as circumstances allow. In our experiment, this means optimizing right down to fractions of a penny. However, the subjects seem to see no contradiction between such optimizing and "fair" behavior, since the median subject reports as fair pretty much what actually happened towards the end of the games that he or she played. These results are consistent with a view that regards behavior as being shaped by social norms in the minds of the subjects, but which sees the social norms themselves being determined by evolutionary considerations of which the subjects are only dimly aware.

However, we do not think it appropriate to make any wide claims for game theory as a predictor of human behavior, in spite of what we regard as the remark- 
able sharpness of our results. The reason is that we are vulnerable to the criticism that we made the process of "myopic adjustment" focal by featuring it in our graphic display ${ }^{14}$. One reply to this criticism is to note the similarity between the results in Case $1 \mathrm{E}$ and Case 2, even though the expected utility indicator was absent in the second situation. However, the important point is much less tendentious.

We do not argue that social norms that isolate particular focal points are unimportant in determining how people behave both inside and outside the laboratory. This paper is about how social norms get established and extinguished. In particular, we believe that it is a major error to suppose that social norms are commonly so rigid that they are able to sustain behavior in the long run that is not in equilibrium. Our experiment shows that it is relatively easy to displace whatever norms our subjects brought into the laboratory by norms that are consistent with an optimizing scenario.

\section{Appendices}

\section{A1 Instructions}

\section{Bargaining Experiment}

In this experiment, you will bargain via the computing equipment in front of you with the people seated at the the other machines in the room. You will participate in a large number of very short bargaining sessions. Whether you are player I or player II in these sessions is determined randomly. Sometimes you will be player I and sometimes player II. After each session, you will be randomly paired with a new bargaining partner.

In each bargaining session, you and your counterpart for that session will have the opportunity to split a "cake" between you. The cake will be represented by a blue region on your monitor screen. You will each simultaneously make a claim. If the pair of claims made by you and your counterpart lies well within the blue region on your screen, then you each get your claims. If the pair of claims lies well outside the blue region, you both get nothing in that session. If the pair of claims lies close to the boundary of the blue region, then the computer will sometimes allow the claims and sometimes it will disallow them. The closer the pair of claims is to the boundary of the blue region, the less likely the computer is to find them acceptable.

${ }^{14}$ We do not accept that such criticism can be neglected because it involves a "Catch 22 ". Nobody anticipates that subjects would optimize if they were not provided with information in an easily digestible form that indicates what optimal behavior is. The catch is that the necessary information cannot be provided without focusing attention on what is optimal. 
You will be bargaining for lottery tickets. After every ten bargaining sessions, each player may possibly win $\$ 10$. Each lottery ticket that you acquired during the preceding ten bargaining sessions gives you one chance of a win. How many lottery tickets you get during the bargaining will depend partly on chance. If you bargain so as to maximize the number of lottery tickets that you would get on average, this will make the probability of winning $\$ 10$ largest. Since you will take part in forty sessions in all, you will have four separate opportunities of winning $\$ 10$. If you are very lucky, you may therefore win $\$ 40$ on top of your participation free. But please do not complain if you win nothing at all. When things are done at random, it is unavoidable that some people will be unlucky.

After the bargaining sessions are over, you will be asked to complete a computerized questionnaire. When all subjects have completed the questionnaire, the computer will display how much we owe you. The amount will include your $\$ 2$ attendance fee, and any money you won during the experiment. Please remain in your seat until the supervisor calls your seat number and then bring your seat tag so that you can be paid.

This is not an experiment to find out what kind of person you are. When we see the results, we shall neither know nor care who did what. We are only interested in what happens on average. So please don't feel that some particular sort of behavior is expected of you. However, we do ask that you do not talk to the other subjects or look at their screens. It is important to the experiment that our subjects interact only via the computer equipment.

Now press the SPACE BAR on your keyboard. You will see a demonstration that will review the information in these instructions and give you hands-on experience of how claims are made. Remember to keep pressing the SPACE BAR to see a new screen. There is no need to hurry. You may have to wait for the other subjects to be ready anyway. If you still have questions after seeing the demonstration, there will then be an opportunity to ask the supervisor. 


\section{A2 Tables and Figures}

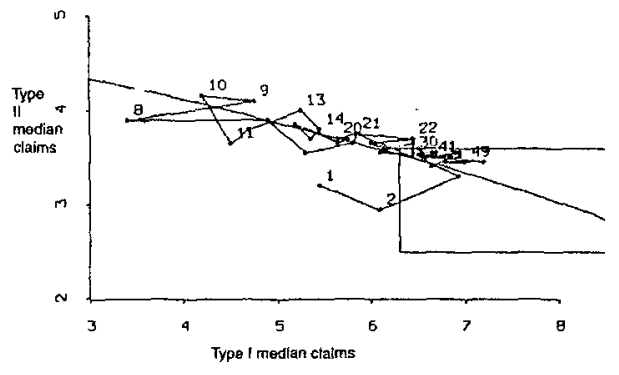

Figure Al(a): Case 1E, Expt. 1

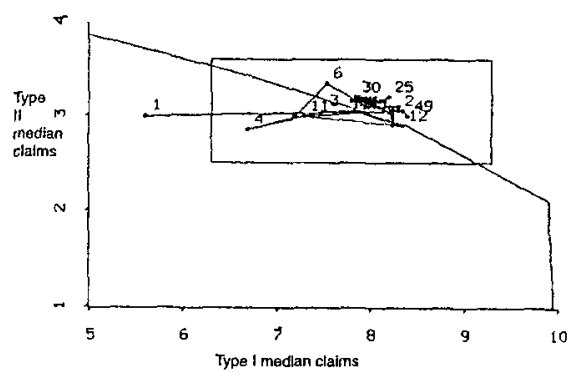

Figure A1(c) : Case iN, Expl. 11

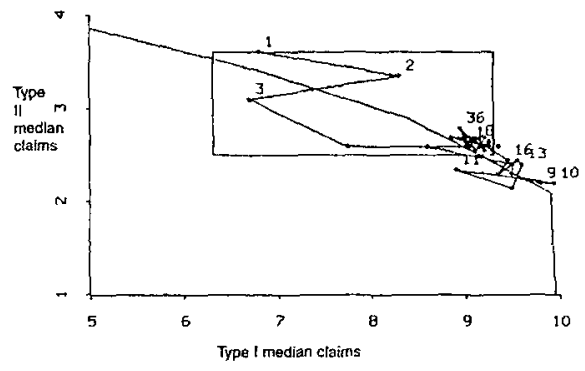

Figure A1(e) : Case 1U, Expt. 13

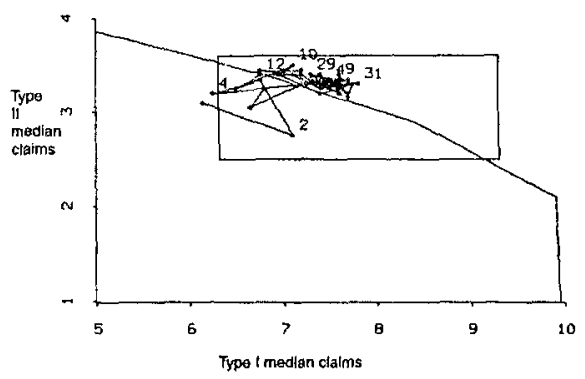

Figure A1(b) : Case 1k, Expt. 8

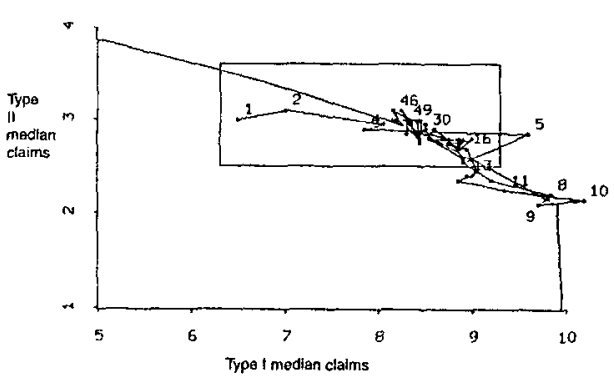

Figure A1(d): Case 1U, Expl. 12

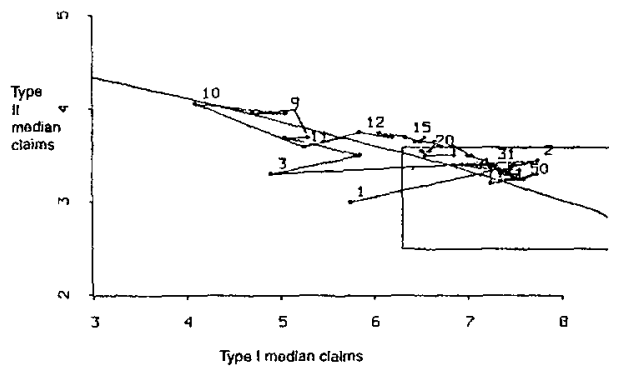

Flgure A1(f) : Case 2x Expt. 17

Fig. A1. Trajectories of median claims for selected experiments 


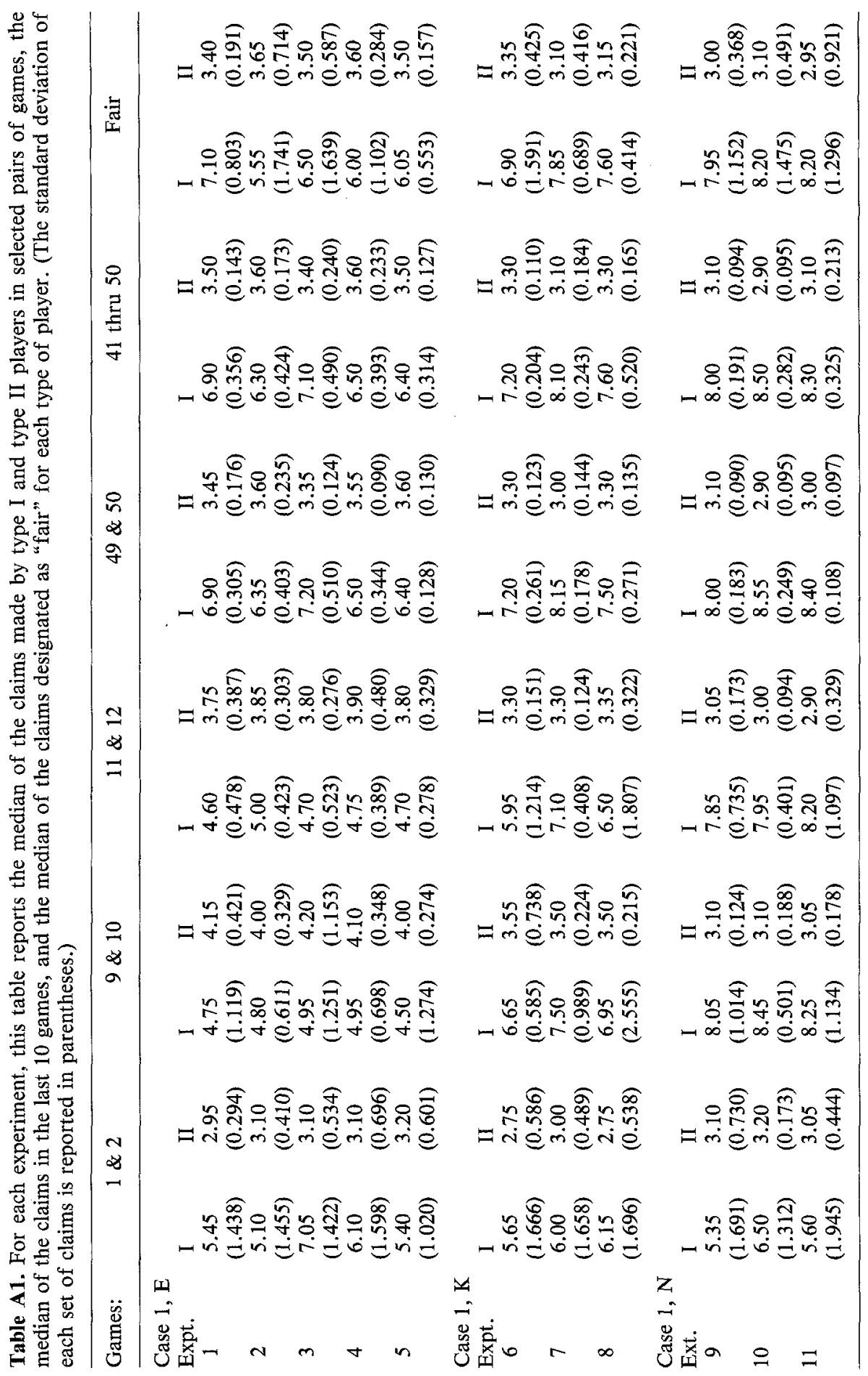




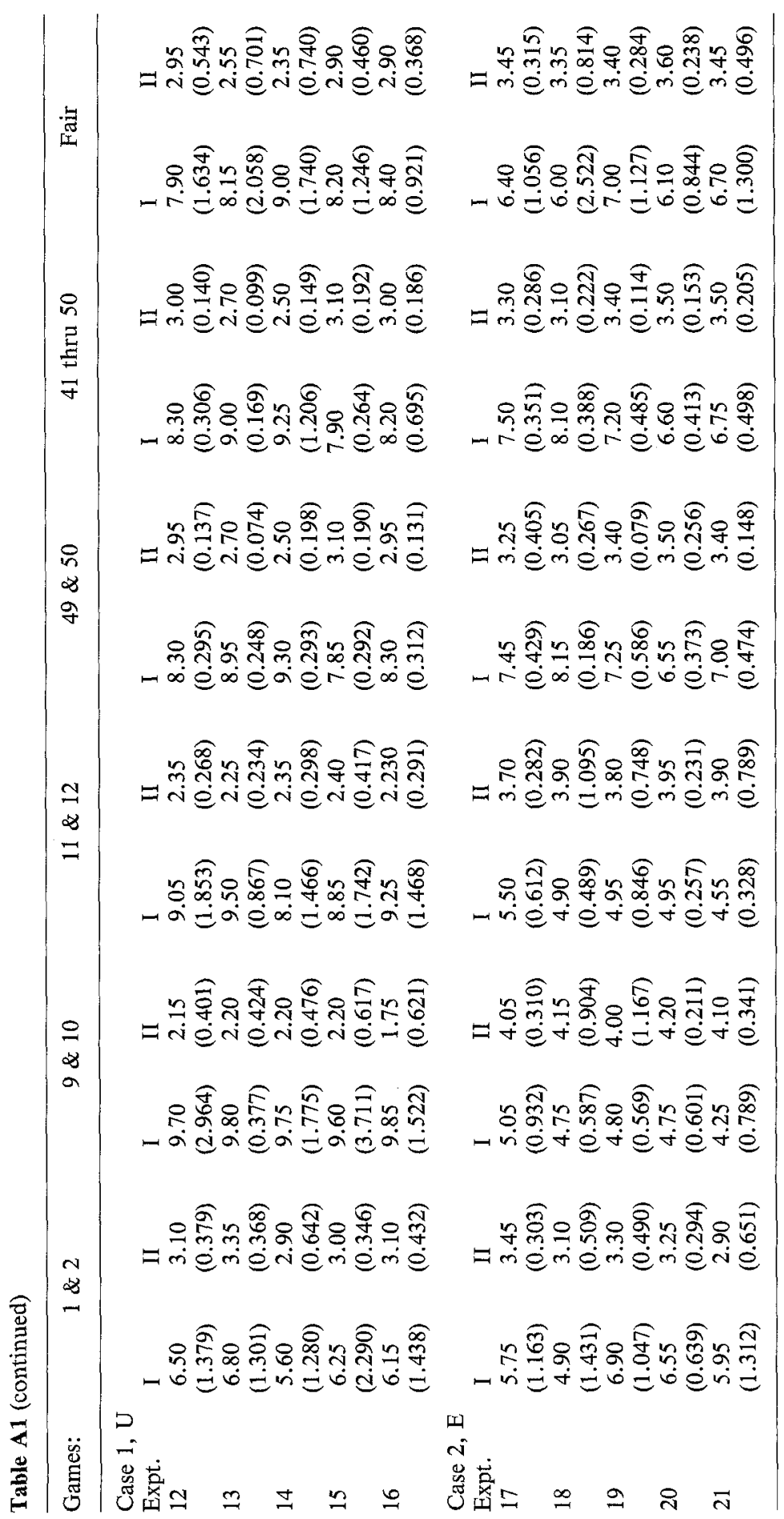


Table A2. Summary statistics describing the median type I and type II claims reported in Table A1 for each treatment and selected pairs of games

\begin{tabular}{|c|c|c|c|c|c|}
\hline Games & Mean & $\begin{array}{l}\text { Standard } \\
\text { deviation }\end{array}$ & Mean & $\begin{array}{l}\text { Standard } \\
\text { deviation }\end{array}$ & $\begin{array}{l}\text { Correlation } \\
\text { coefficient }\end{array}$ \\
\hline \multicolumn{6}{|c|}{ Case $1, E, 5$ experiments } \\
\hline & \multicolumn{2}{|c|}{ Type I claims } & \multicolumn{3}{|c|}{ Type II claims } \\
\hline $9 \& 10$ & 4.790 & 0.185 & 4.090 & 0.089 & 0.634 \\
\hline $11 \& 12$ & 4.750 & 0.150 & 3.820 & 0.057 & 0.585 \\
\hline $49 \& 50$ & 6.670 & 0.367 & 3.510 & 0.108 & -0.997 \\
\hline \multicolumn{6}{|c|}{ Case $1, K, 3$ experiments } \\
\hline & \multicolumn{2}{|c|}{ Type I claims } & \multicolumn{2}{|c|}{ Type II claims } & \\
\hline $9 \& 10$ & 7.033 & 0.431 & 3.517 & 0.029 & -0.770 \\
\hline $11 \& 12$ & 6.517 & 0.575 & 3.317 & 0.029 & -0.025 \\
\hline $49 \& 50$ & 7,617 & 0.486 & 3.200 & 0.173 & -0.951 \\
\hline \multicolumn{6}{|c|}{ Case $1, N, 3$ experiments } \\
\hline & \multicolumn{2}{|c|}{ Type I claims } & \multicolumn{2}{|c|}{ Type II claims } & \\
\hline $9 \& 10$ & 8.250 & 0.200 & 3.083 & 0.029 & 0.000 \\
\hline $11 \& 12$ & 8.000 & 0.180 & 2.983 & 0.076 & -0.999 \\
\hline $49 \& 50$ & 8.317 & 0.284 & 3.000 & 0.100 & -0.967 \\
\hline \multicolumn{6}{|c|}{ Case $1, \mathrm{U}, 5$ experiments } \\
\hline & \multicolumn{2}{|c|}{ Type I claims } & \multicolumn{2}{|c|}{ Type II claims } & \\
\hline $9 \& 10$ & 9.740 & 0.096 & 2.100 & 0.197 & -0.627 \\
\hline $11 \& 12$ & 8.950 & 0.533 & 2.330 & 0.057 & -0.617 \\
\hline $49 \& 50$ & 8.540 & 0.578 & 2.840 & 0.238 & -0.995 \\
\hline \multicolumn{6}{|c|}{ Case 2, E, 5 experiments } \\
\hline & \multicolumn{2}{|c|}{ Type I claims } & \multicolumn{2}{|c|}{ Type II claims } & \\
\hline $9 \& 10$ & 4.720 & 0.291 & 4.100 & 0.079 & -0.218 \\
\hline $11 \& 12$ & 4.970 & 0.340 & 3.850 & 0.100 & -0.772 \\
\hline $49 \& 50$ & 7.280 & 0.591 & 3.320 & 0.175 & -0.972 \\
\hline
\end{tabular}


Table A3. For selected pair of games in each experiment, this table reports the maximum expected gain that a player can achieve by deviating from the median claim for his type that was reported in Table A1 when the player expects his opponent to make the corresponding median claim that was reported for her type in Table A1. The gain is the maximum of that achievable by player I and player II. The numbers assigned to each experiment are the same as those assigned in Table A1. (The numbers in parentheses are the median and 90th percentile of the maximum expected gains obtained by deviating optimally from each of the type I or type II claims made in the designated pair of games. ${ }^{*}$ The maximum expected gain in this case is calculated assuming that a claim is matched randomly against one of the 12 opposing claims actually made in the designated games.)

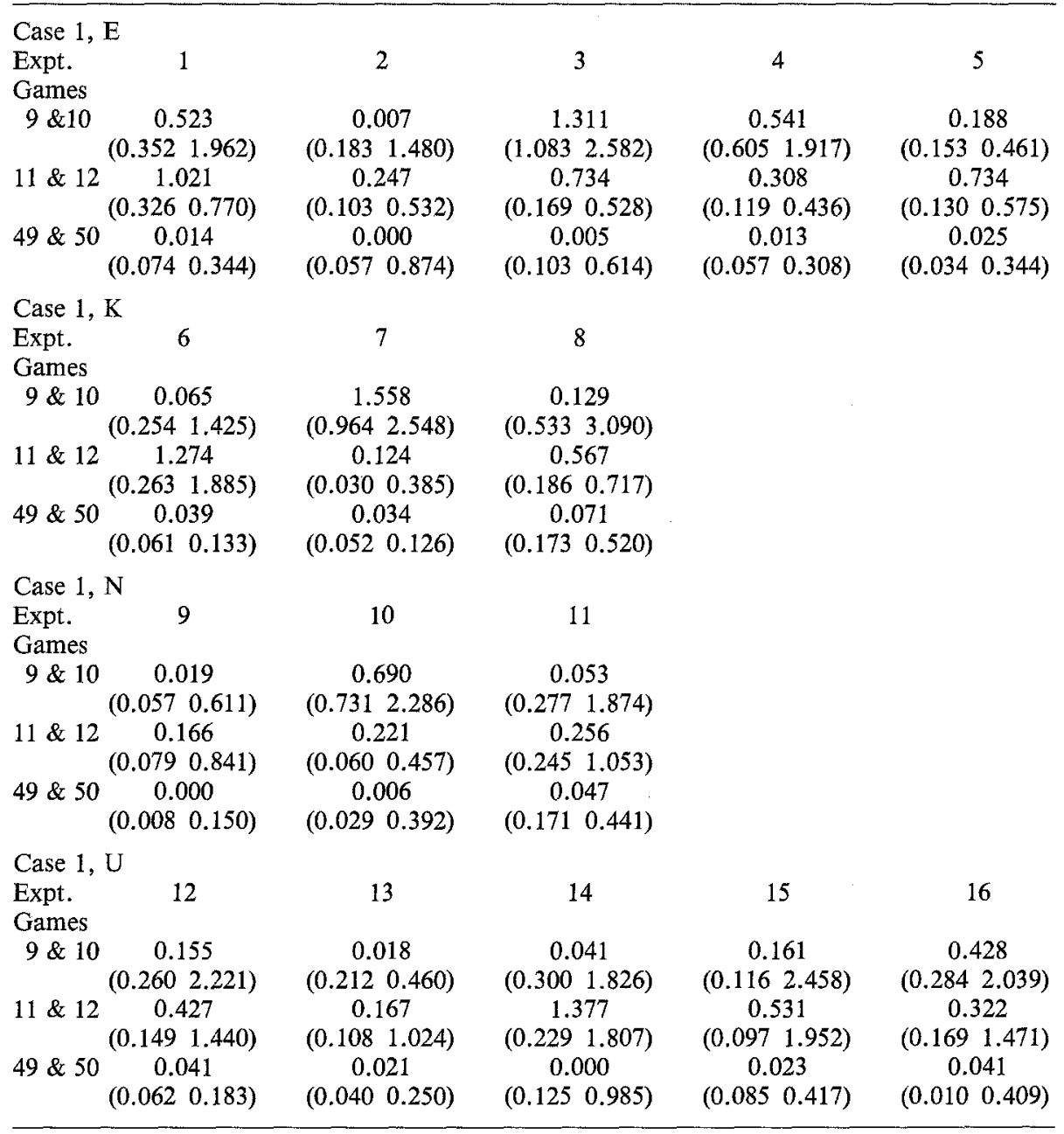


Table A3 (continued)

\begin{tabular}{|c|c|c|c|c|c|}
\hline \multicolumn{6}{|c|}{ Case 2, E } \\
\hline Games & & & & & \\
\hline $9 \& 10$ & $\begin{array}{c}0.404 \\
(0.228 \quad 1.614)\end{array}$ & $\begin{array}{c}0.523 \\
(0.514 \quad 1.771)\end{array}$ & $\begin{array}{c}0.007 \\
(0.523 \quad 2.210)\end{array}$ & $\begin{array}{c}0.866 \\
(0.650 \quad 1.481)\end{array}$ & $\begin{array}{c}0.093 \\
(0.107 \quad 0.871)\end{array}$ \\
\hline $11 \& 12$ & $\begin{array}{c}0.307 \\
(0.1210 .786)\end{array}$ & $\begin{array}{c}0.159 \\
(0.245 \quad 1.175)\end{array}$ & $\begin{array}{c}0.484 \\
(0.2081 .255)\end{array}$ & $\begin{array}{c}0.017 \\
(0.0330 .550)\end{array}$ & $\begin{array}{c}0.508 \\
(0.225 \quad 1.406)\end{array}$ \\
\hline $49 \& 50$ & $\begin{array}{c}0.003 \\
(0.118 \quad 0.608)\end{array}$ & $\begin{array}{c}0.004 \\
(0.043 \quad 0.229)\end{array}$ & $\begin{array}{c}0.130 \\
(0.1890 .765)\end{array}$ & $\begin{array}{c}0.043 \\
(0.122 \quad 0.613)\end{array}$ & $\begin{array}{c}0.000 \\
(0.077 \quad 0.373)\end{array}$ \\
\hline
\end{tabular}

* There are 24 type I or type II claims and, hence, 24 maximum expected gains associated with each pair of games and each experiment. The median is the average of the 12th and 13th largest maximum expected gains. The 90th percentile is the average of the $3 \mathrm{rd}$ and 4 th largest expected gains

\section{References}

[1] Binmore K, Dasgupta P (1987) The economics of bargaining. Basil Blackwell, Oxford

[2] Binmore K, Morgan P, Shaked A, Sutton J (1991) Do people exploit their bargaining power: An experimental study. Games and Economic Behavior 3:295-322

[3] Cooper J, DeJong D, Forsythe R, Ross T (1991) Selection criteria in coordination games: Some experimental results. American Economic Review 80:218-233

[4] Gibbons J, Chakraborti S (1992) Nonparametric statistican inference. 3rd edition Marcel Dekker Inc. New York

[5] Harsanyi J (1977) Rational behavior and bargaining equilibrium in games and social situations. Cambridge University Press, Cambridge

[6] Isbell J (1960) A modification of Harsanyi's bargaining model. Bulletin of the American Mathematical Society $66: 70-73$

[7] Kalai E (1977) Proportional solutions to bargaining situations: Interpersonal utility comparisons. Econometrica $45: 1623-1630$

[8] Kalai E, Smorodinsky M (1975) Other solutions to Nash's bargaining problem. Econometrica $45: 1623-1630$

[9] Myerson R (1977) Two-person bargaining and comparable utility. Econometrica $45: 1631-1637$

[10] Nash J (1950) The bargaining problem. Econometrica 18:155-162

[11] Peters H (1986) Bargaining game theory. PhD thesis, Proefschritt Universitat Nijmegen

[12] Raiffa H (1953) Arbitration schemes for generalized two-person games. In: Kuhn H, Tucker A (editors) Contributions to the Theory of Games II, Princeton University Press, Princeton

[13] Rao C (1973) Linear statistical inference and its applications. 2nd edition. Wiley, New York

[14] Rawls J (1972) A theory of justice. Oxford University Press, Oxford

[15] Roth A (1979) Axiomatic models of bargaining. Springer-Verlag, Berlin

[16] Schelling T (1960) The strategy of conflict. Harvard University Press, Cambridge, Mass.

[17] Van Huyck J, Battalio R, Beil R (1991) Strategic uncertainty, equilibrium selection principles and coordination failure. American Economic Reviews 80:234-238

[18] Van Huyk J, Battalio R, Mathur S, Ortmann A, Van Huyck P (1991) On the origin of convention: Evidence from symmetric bargaining games. Working Paper, Economics Department, Texas A\&M University 